\section{Self-paced and irregular methods of subjective estimation of pain}

JUDITH ANN MENZER and RICHARD P. SMITH, University of Louisville, Louisville, Ky. 40208, and LEE S. CALDWELL, U.S. Amy Medical Research Laboratory, Fort Knox, Ky. 40121

Sixteen Ss estimated the pain produced by isometric muscle contraction maintained to exhaustion. Two loads (25\% and 40\%), two scales (5-and 10-point), and two scaling methods (self-paced and irregular) were used. Pain levels were reported earlier with the $40 \%$ load $(p<.01)$, with the self-paced method $(p<.01)$, and with the 5-point scale $(p<.01)$. Both the 10-point scale and the irregular reporting method produced longer response times at the terminal intensities $(p<.01)$.

Caldwell (1967) and Caldwell \& Smith (1967) have shown that a simple technique may be used to assess the subjective components of performance decrement (fatigue) produced by isometric muscle contractions. They obtained essentially linear functions relating the intensity of noxious sensations to contraction time. This may reflect a linear growth of the sensations, as concluded by the authors and by Hosman (1967), but it is also possible that the linearity may be due either to a tendency of $S$ to estimate his endurance at the beginning of the performance and divide the estimated total time into the appropriate number of parts, or simply to repeat the first temporal interval.

A method devised by G. M. Smith et al (1966) and first reported by Beecher (1966) offered an opportunity for a test of Caldwell and Smith's conclusion as this technique requires $S$ to report his sensations of pain at irregular, unpredictable intervals so there should be no tendency to concentrate on time rather than on the sensations. The primary purpose of the present paper was to compare the self-paced me thod of Caldwell and Smith with the irregularly paced method of G. M. Smith et al.

SUBJECTS AND APPARATUS

Sixteen male college students served as Ss. Their ages ranged from 18 to 24 years with a mean of 19.8 years. The apparatus was an adjustable isometric hand dynamometer with strain gauges, a strain amplifier, an ink-writing recorder, and a voltmeter with a scale marked in pounds which served as a display for $\mathrm{S}$. The apparatus has been described in detail elsewhere (Caldwell, $1967)$.

\section{PROCEDURE}

Prior to the experimental sessions Ss were given practice in the scaling procedures to be used in the study. After grip strength was determined for both hands, $S$ was told to take the dynamometer in his left hand and to squeeze just hard enough to turn on the indicator lamp and to keep it on for as long as possible. This lamp, which was mounted on the display meter and controlled by an adjustable relay, was set to illuminate when the applied force equalled half S's grip strength. Ss received practice with four procedures-reporting the time of appearance of the various pain intensities using both 5 - and 10-point scales, and estimating the intensity of the sensation on both scales when interrupted on a random schedule. Interruption times of $9,16,26,32,40,48$, $53,59,66,75,85,93,100,105,112,120$, $128,134,142$, and 148 sec were determined by drawing at random from the numbers 5 through 10 and adding the result to the previous total. Ss were told they could terminate performance if the pain became intolerable.

In the experiment proper there were eight conditions formed by the factorial combination of two loads $(25 \%$ and $40 \%$ of maximum strength) with two scales (5- and 10-point) and two scaling methods (selfpaced and irregular). The eight experimental combinations presented in a counterbalanced order were administered in two sessions of four trials each. Hands were alternated to reduce fatigue. Ten-minute rests were given between trials. RESULTS AND DISCUSSION

The data were treated by an analysis of variance for repeated measures. To compare the results for the two scales only the scores for points $2,4,6,8$, and 10 of the 10 -point scale were used in the analysis. The significant effect of loads $(F=11.29$, $\mathrm{df}=1 / 15, \mathrm{p}<.01)$ indicates that with an increase in load from $25 \%$ to $40 \%$ of maximum strength there was a general tendency to report the pain levels earlier in the performance. It may be seen in Figs. 1 and 2 that every intensity was reported sooner for the $40 \%$ than for the $25 \%$ load, demonstrating the sensitivity of the scaling to contraction strength. A significant interaction between Loads and Intensities $(F=14.11, \mathrm{df}=4 / 60, \mathrm{p}<.01)$ indicated that increasing the load did not produce a uniform decrease in the contraction time required to induce the various pain levels. That is, increasing the load simply increased the slope of the pain function without altering the $\mathrm{Y}$-intercept, thus producing the greatest change at the highest pain intensity. There was no indication from the statistical analysis that the magnitude of the load effect was influenced either by the size of the scale interval or by the method of reporting.

The scaling method had a small (mean difference of $2.6 \mathrm{sec}$ ) but significant effect $(F=12.89, \mathrm{df}=1 / 15, \mathrm{p}<.01)$ on the times at which the pain levels were reported. The various levels of pain were reported earlier when $S$ was free to announce their times of appearance than when he was required to

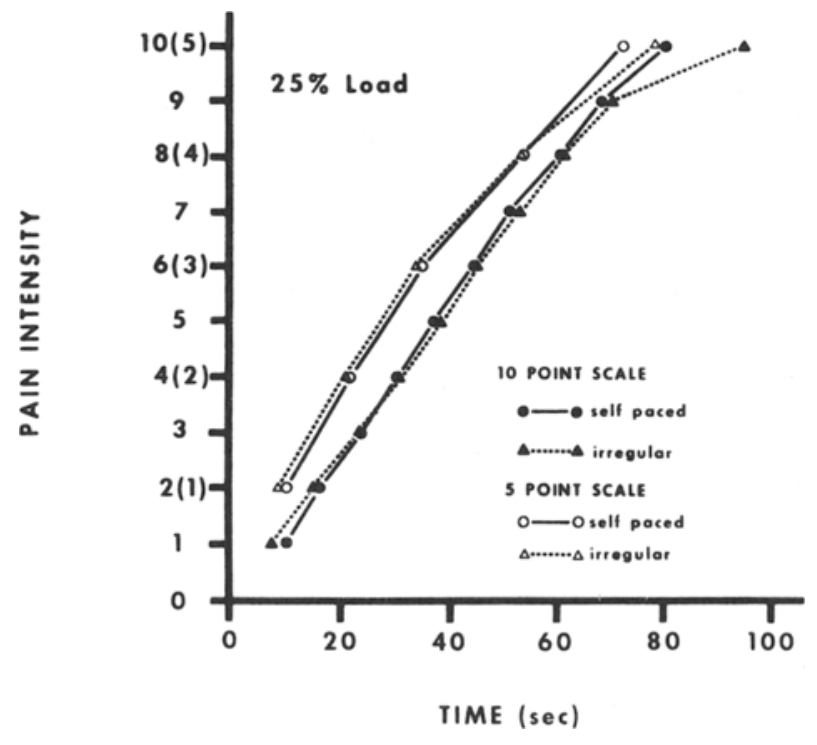

Fig. 1. Pain ratings for a 25\% maximum load for both 5-point and 10-point scales using self-paced and irregular ( $E$ paced) methods of reporting. 
estimate the intensity upon demand. Figures 1 and 2 show that the reporting method had its greatest effect at the terminal intensities; that is, there was a significant interaction of Methods and Intensities ( $F=15.50$, $\mathrm{df}=4 / 60, p<.01)$. This terminal effect may have been due to a reluctance of $S$ s to terminate performance until $E$ called for a report, though $S s$ were told they could call out the number corresponding to the final level and stop when the pain became too intense. The difference between the methods may also reflect the influence of E on S's performance. That is, the personal interaction between $E$ and $S$ in the one procedure may have increased the motivation of $S$ and thus delayed his pain reports.

The data obtained with the 5- and 10-point scales were compared and it was found that all pain intensities were reported sooner on the coarse scale. This difference $(\mathrm{F}=13.07, \mathrm{df}=1 / 15, \mathrm{p}<.01)$ may merely reflect the fact that a 10-point scale is more difficult to use than a 5-point scale and hence requires more time. At the present time there is insufficient data to determine precisely what influence the coarseness of the scale has on the ratings of a sensation, but these results indicate the presence of a substantial effect attributable to the scale unit size independent of the range of sensations judged.

The primary concern in this study was the comparison of two rating methods. The comparability of the pain functions yielded by the two methods suggests that the linearity of the curves previously obtained with the self-report procedure was not due to $S$ f fractionating time, because essentially linear functions were obtained even with irregular reporting times selected by $E$. The comparatively long interval between the last two scale points may represent an end spurt, or a tendency to assess reserve incorrectly. It may be, too, that for these comparatively light loads and the longer contraction times fatigue was attenuated by recovery phenomena. Both of these loads were well below the value at which internal pressure in the muscle occludes the blood supply (Humphreys \& Lind, 1963; Caldwell, 1967) so that some recovery was possible during the performance. The differences attributable to the main effects of me thods, though statistically significant, were small. Thus, there is no basis at the present time for saying that one method is better or more orderly for scaling pain other than on the basis of difficulties introduced by the active participation of $E$ in the rating procedure.

\section{REFERENCES}

BEECHER, H. K. Pain: One mystery solved Science, 1966, 151,840-841.

CALDWELL, L. S. The scaling of effort produced by strenuous isometric muscle contractions. USAMRL Report No. 749, 1967.

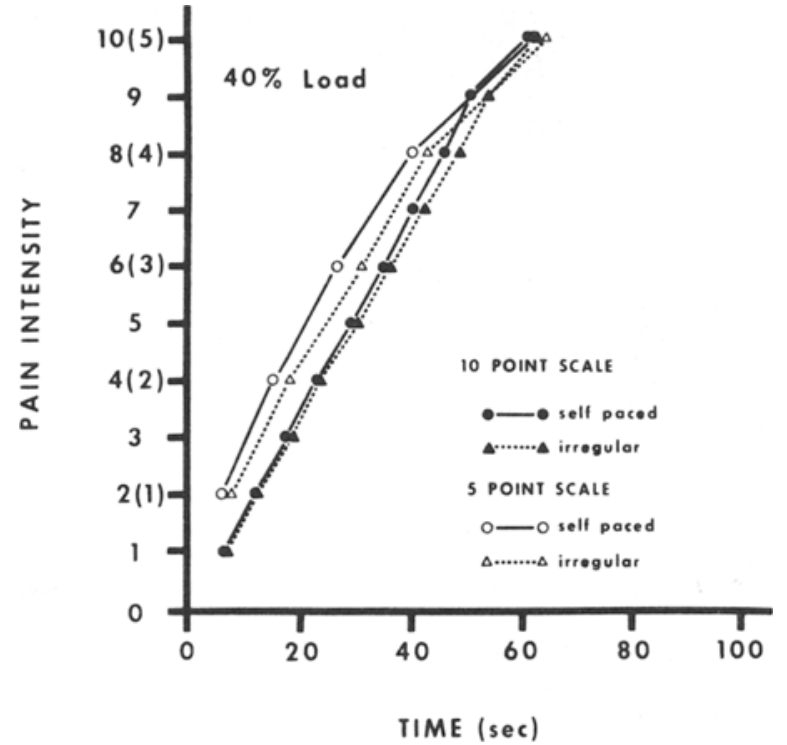

Fig. 2. Pain ratings for a $40 \%$ maximum load for both 5-point and 10-point scales using self-paced and irregular ( $E$ paced) methods of reporting.

CALDWELL, L. S., \& SMITH, R. P. Subjective estimation of effort, reserve, and ischemic pain. USAMRL Report No. 730, 1967.

HOSMAN, J. Adaptation to muscular effort. Report of the Psychological Laboratory, University of Stock holm, No. 223, 1967.

HUMPHREYS, P. W., \& LIND, A. R. The blood flow through active and inactive muscles of the forearm during sustained hand-grip contrac- tions. Journal of Physiology, 1963, 166, 120-135.

SMITH, G. M., EGBERT, L. D., MARKOWITZ, R. A., MOSTELLER, F., \& BEECHER, H. K. An experimental pain method sensitive to morphine in man: The submaximum effort tourniquet technique. Journal of Pharmacology \& Experimental Therapeutics, 1966, 154, 324-332.

\section{Bidirectional associations in multi-trial free recall}

RICHARD C. ANDERSON and GRAEME H. WATTS, University of Illinois, Urbana, Ill. 61801

An augmented measure of organization in free recall, combining reverse-order intertrial repetitions (R-ITR) and the usual same-order intertrial repetitions (S-ITR), was more highly correlated with measures of organization and number of words recalled than a measure including S-ITRs alone. The conditional probabilities of an S-ITR or an $R-I T R$ between one pair of trials given an $R-I T R$ between another pair of trials and the conditional probabilities of an S-ITR or an $R-I T R$ given an S-ITR were well above chance. While in every case the observed proportion of $S$-ITRs was greater than the proportion of R-ITRs, it is estimated. excluding $S s$ who alphabetized the entire list, that the majority of the observed intertrial repetitions could be explained in terms of bidirectional associations.

The intertrial repetition is a commonly used index of organization in free recall. We shall refer to the event usually investigated as a same-order intertrial repetition (S-ITR) since it involves pairs of words recalled (1) adjacent to one another, and (2) in the same order on two occasions. For example, if a recall protocol contains NECKLACE, SANDAL on two occasions, this is an S-ITR However, the appearance of NECKLACE, SANDAL on one occasion and SANDAL. NECKLACE on another is not customarily counted. The latter event is an instance of a reverse-order intertrial repetition (R-ITR). There are perfectly good theoretical reasons for including R-ITRs in the measure of organization. The R-ITR can be conceived as representing a bidirectional association and recall could be organized in part in terms of bidirectional associations. The purpose of 